\title{
The power of suggestion: should authors nominate peer reviewers?
}

\author{
David P. Cann ${ }^{1, *}$ (D) and Christopher F. Blanford ${ }^{2, *}$ (D) \\ ${ }^{1}$ School of Mechanical, Industrial and Manufacturing Engineering, Oregon State University, Corvallis, OR 97331, USA \\ ${ }^{2}$ School of Materials and Manchester Institute of Biotechnology, University of Manchester, 131 Princess Street, Manchester M1 7DN, \\ UK
}

Received: 11 December 2017
Published online:
19 December 2017
C) Springer Science+Business
Media, LLC, part of Springer
Nature 2017

Soliciting highly qualified reviews is one of the greatest challenges facing the scientific peer review process. In 2017, the Journal of Materials Science will have received over 8000 submissions. Around two-fifths of these go out for review, each requiring 4-6 invitations to ensure enough usable reports to take action on the manuscript. This works out at more than 16000 invitation emails per year. Certain times of the year are particularly problematic: we get our biggest increase in submission rate in July, right before many researchers (and thus reviewers) go away on vacation. We editors work hard to be fair and prompt in our decisions, but there's evidence in some fields that submissions for which editors have a harder time getting peer reviews are reviewed less favorably [1]. As a result, many journals, including this one, ask their contributors to suggest suitable referees for their work.
Over the last year, however, the Journal of Materials Science has conducted a small experiment in our submission process. We stopped enforcing our requirement that authors provide the names, affiliations, and email addresses of at least three suitable referees, together with a brief justification of their suitability. There is no consensus among our peer journals about requiring a list of suggested reviewers, and there have been mixed views about this change among this journal's editors. Here are arguments for both sides from two of this journal's longest-serving editors.

\section{Cann: the case for nominating referees}

From my vantage point, the list of suggested reviewers is an important declaration from the author about the quality of the manuscript and a statement

Address correspondence to E-mail: cann@engr.orst.edu; christopher.blanford@manchester.ac.uk 
of their intent to support the peer review process. As an editor, I look at that list carefully. When the peer review process works best, reviewers make significant contributions to improving the quality of the manuscript. Reviewers are essentially the first line of defense in ensuring that the manuscript is technically sound and that the conclusions are fully supported by the evidence. Reviewers can also help improve the technical aspects of the manuscript by suggesting alternate explanations or analyses, or proposing additional experiments that would strengthen the conclusions.

Of course, it is unrealistic to expect that every article published in the Journal of Materials Science, or frankly any journal, will reap the full benefits of contributions from meticulous, thoughtful reviewers. It is incredibly difficult to obtain high-quality reviews for every manuscript. However, I believe getting the most out of the peer review process is a goal that we should strive for. This requires enlisting the best reviewers possible, with the right expertise. This is where the list of suggested reviewers is vital. The authors should know the context of their work and should know who would best serve as reviewers of that work.

The list of suggested reviewers be can interpreted two ways: as either a genuine statement of the authors desire to have their manuscript meaningfully improved by the thoughtful, constructive critique of their peers, or as a list of reviewers most likely to recommend publication as rapidly as possible. It is not always easy to tell the difference, but tools like Web of Science and Google Scholar can help provide insights into a potential reviewers' appropriateness for the manuscript in question. The suggestion of former students or advisors, or people unrelated to our discipline is obviously not judged favorably. (An author of a manuscript I was handling recommended an Agricultural Economist!) Researchers that are visible research leaders or major contributors to the field are judged more favorably.

At the Journal of Materials Science, each editor handles suggested reviewers differently. I typically only actually use one suggested reviewer on the list, but as I have described above that list has a much greater meaning in the overall context of the evaluation of the manuscript.

One of the other complications I encounter as editor is that this list of suggested reviewers sometimes leads to the issue of frequently requesting reviews from the same people. It is difficult for me to request a review from someone whom I have asked too many times before. However, on one occasion I sent a review request to a colleague at another institution whom I have asked many times before and who no doubt receives requests from many other journals. The email I sent to him included a preamble apologizing for asking him for yet another review. His response was that he would be glad to review the article because, and I quote him directly, "it is our sacred duty." I have never forgotten that phrase.

\section{Blanford: the case against nominating referees}

In March 2017, an observant editor working at a journal of a major scientific publisher contacted me about my name being used suspiciously as a suggested reviewer. There was a plausible Gmail account associated with my name, but the paper covered topics outside my research area. I let the editor know the email account was not mine. The publisher's staff found a dozen similar cases in five journals. Although the culprit admitted his guilt, at the time of this writing, only two have been marked as retracted, four remain online, and four do not appear anywhere. The falsely reviewed papers will remain, with their retractions, a publicly visible record of his unethical behavior, and other journals will scrutinize any work from this author.

Surely the editors should have spotted this, right? However, from their perspective, their editorial management systems would show that the fake Chris Blanford has a record of accepting review invitations and responds before the deadline with useable reports. This sounds great, because an editor gets a response (and not necessarily an acceptance) from only about a third of the total invitations to review a paper. Plus, that editor could easily assume that one of the other editors would have certainly checked that email address was legitimate.

So, do we insist that reviewers use institutional email addresses only? This move would effectively exclude reviewers in places where their university accounts are less reliable than third-party commercial email services. Sometimes, editorial correspondence is filtered by institutional email servers. For example, in September 2017, Shandong University was rejecting emails that were sent from the J. Mater. Sci.'s 
editorial manager servers. A qualified researcher at a company might need to separate his or her peer reviewing contributions from his or her work account. Anecdotally, at least one US university has stated that editorial duties should be kept separate from work email; it wasn't clear whether that extended to contributing as a peer reviewer. There are too many practical problems for a blanket exclusion to apply.

Even with a legitimate email address, there is still the danger of peer review rings, in which groups of academics agree to write favorable reviews of each others' work. This type of fraud is counterproductive to the advancement of science, insidious, and very difficult to police, particularly in our current academic publishing culture where reviewer databases are not widely shared beyond a single publisher's domain. Without a wider connection, how could you connect, say, four author/reviewers colluding with each other if they're publishing in separate journals from separate publishers? The problem is even trickier to avoid positive bias in the softer "he's a good chap" cases, where the author and reviewer are part of the same network (a.k.a., the "old boys' club").

Finally, an author's time needs to be respected. Every corresponding author has faced the over-thetop submission requirements: "Please provide the name, address, telephone number, fax number, email address, mother's maiden name, brief $\mathrm{CV}$, and onepage justification for your choice for each of FIVE suggested referees." This is a (slight) exaggeration, but accurately captures the additional administrative burden that's placed on the creators of the content that the publishers will sell.

The system of scientific communication relies on trust. We trust that authors have actually carried out the measurements they are presenting and that their data are real and not manipulated. We trust that the editors and peer reviewers will be fair and impartial. However, it is still too easy to manipulate the peer review system through suggested reviewers. I am in favor of helping out the busy editors, but it is better for the authors and the scientific record if no reviewers are suggested at all.

\section{Some ways forward}

The solutions all require working on a scale larger than that of individual journals or publishers. Publons maintains a database of peer review reports. The Publons database has been linked to two products from their parent company, Clarivate: the editorial management software ScholarOne and the scientific paper and citation indexing service Web of Knowledge. Integrating these databases, they offer a Reviewer Locator tool. Aries Systems, provider of the Editorial Manager system, offers their Reviewer Discovery tool that suggests potential referees by running an article's title and abstract against the ProQuest Community of Scholars database. Elsevier's EVISE system has a Reviewer Finder that matches a submission to researcher "fingerprints" assembled from the data on their Scopus abstract and citation database.

All three systems are welcome additions, but it is too soon to tell if there are biases hidden in the closed-source reviewer recommendation algorithms or what tricks might be developed to pervert their operation. In the longer term, we could require all referees to have publicly visible ORCID profiles. A referee's suitability can then be readily judged by a researcher's experience and his or her publication and peer review track record. We do not yet have enough data to make this feasible, but as more and more journals require valid, publicly visible ORCID profiles for their authors [2], the better our chances are of having a set of publication and review data held by an impartial third party. Once tools like these are in place, our initial debate become moot: we will already have selected our reviewers, indirectly, through our individual contributions as authors and peer reviewers.

The peer review process underpins almost everything in the world of scientific communications. For it to function as effectively as possible, we all must contribute (no matter which mechanism is used to identify and select peer reviewers). Nonetheless, we have to believe that each constructive review helps drives the machinery behind-the-scenes toward quality science. 


\section{References}

[1] Fox CW (2017) Difficulty of recruiting reviewers predicts review scores and editorial decisions at six journals of ecology and evolution. Scientometrics 113:465-477
[2] Carter CB, Blanford CF (2017) All authors must now supply ORCID identifiers. J Mater Sci 52:6147-6149. https://doi.org/ 10.1007/s10853-017-0919-7 